\title{
Mice guide human drug trial
}

\section{Parallel approach to cancer study provides genetic insights.}

\section{BY HEIDI LEDFORD}

$\mathrm{T}$ he mouse hospital at the Dana-Farber Cancer Institute in Boston, Massachusetts, boasts resources rivalling those offered to human patients, including state-ofthe-art medical-imaging facilities, round-theclock nursing staff and access to cutting-edge cancer drugs. The goal is not to cure mice, of course, but to gain insight into clinical trials of those drugs in humans.

The roughly 5,000 rodent residents at DanaFarber are pioneers in an approach known as a co-clinical trial, in which a human clinical trial is partnered with animal studies designed to reveal clues about why some patients respond to a treatment and others fail to do so. The first findings from the Dana-Farber project, published online on $18 \mathrm{March}$ (Z. Chen et al. Nature http://doi.org/hrr; 2012), suggest that adding an experimental drug called selumetinib to standard chemotherapy may help to shrink lung tumours in patients with mutations in a gene called KRAS. The parallel study in the mouse revealed, however, that the drug may fail in those who also carry a mutation in a tumour-suppressor gene called LKB1.

"Normally, very little scientific interrogation occurs during the conduct of a human cancer trial," says David Tuveson, a cancer researcher at the University of Cambridge, UK, who was not involved in the study. "This is a logical and badly needed path forward."

It is standard for new drugs to undergo animal studies before they reach the clinic. But early-stage animal trials can be misleading because they often fail to mimic the conditions of subsequent human trials, says Andrew Kung, an oncologist at Dana-Farber and an author on the mouse study. By contrast, a co-clinical trial is designed to closely match a parallel trial in human subjects.

Selumetinib, developed by Array BioPharma of Boulder, Colorado, and London-based AstraZeneca, is a member of a hotly pursued class of cancer drug that inhibits a crucial biochemical step promoting tumour-cell proliferation and survival. Full results of the human trial, which enrolled 87 patients with KRAS mutations, have yet to be published. Array BioPharma announced last year that, when given with standard chemotherapy, the drug slowed the progress of the cancer. Effects on overall patient survival were not statistically significant.

\section{$\rightarrow$ NATURE.COM For ways to fix trial, see: go.nature.com/wy6bns}

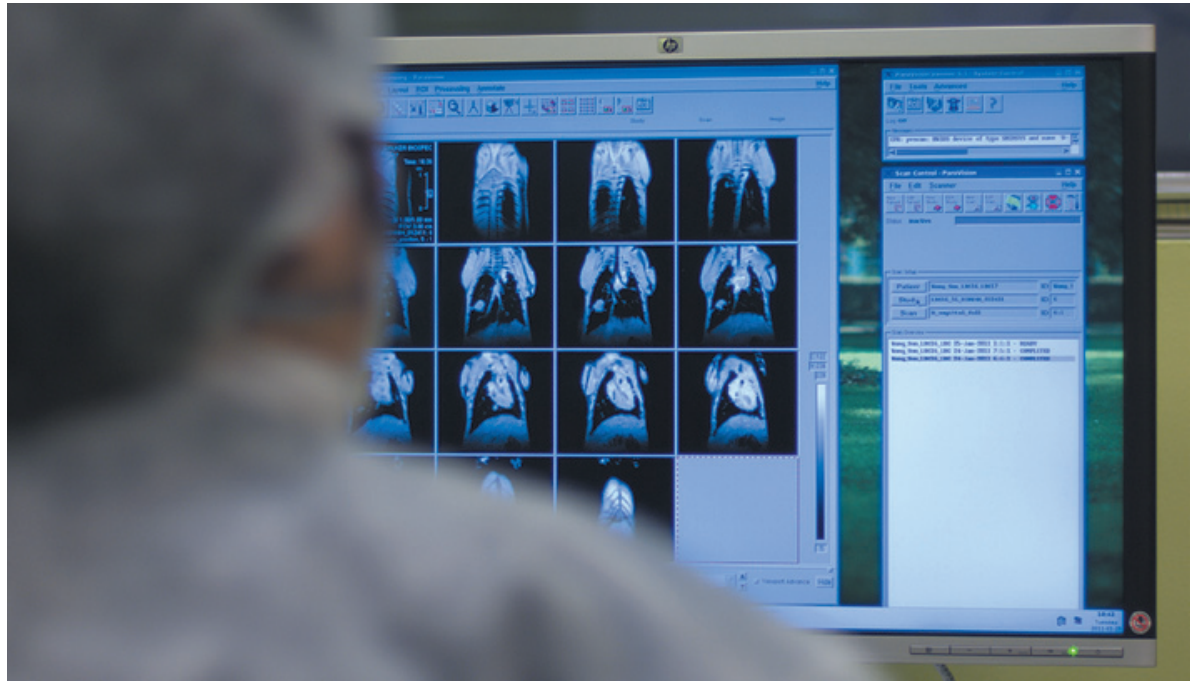

A genetically engineered mouse lung-cancer model offers clues to its human analogue.

Like the human subjects, the hundreds of mice used for the co-clinical trial carried a mutated form of Kras, but some also contained mutations in the tumour-suppressor genes p53 and Lkb1. Mice treated with selumetinib and standard chemotherapy benefited if they had the additional mutation in $p 53$ alone, but the $L k b 1$ mutation seemed to render the drugs ineffective. Outcomes of the mouse trials were measured using methods similar to those for the human subjects, which meant relying on non-invasive imaging techniques, rather than simply killing the ani-

"It is a new way to use mice as a looking glass into the human condition." mals and measuring tumour volume, as is often done in animal studies.

Overall, the mouse study suggests that, in future trials of selumetinib, patients should be screened for the presence of $L K B 1$ mutations, says Kung. That would allow researchers to determine whether a genetically identifiable subset of patients resistant to the drug may be affecting their results.

The results in mice also suggest that an imaging technique called FDG-PET can measure short-term tumour responses to selumetinib. The method could be a boon for human studies, because patients generally cannot be subjected to repeated tumour biopsies.

The co-clinical approach could speed up drug development as oncology increasingly embraces complex treatment regimens involving multi-drug cocktails and tailored for specific genotypes, says Hugues de Thé, a cancer researcher at the Paris Diderot University. He has used a similar approach in a trial of drugs for a form of leukaemia, but says that the new work is a "technical tour de force" because of the genetic complexity of the tumours studied.

Many other centres are developing facilities for co-clinical trials, but the experiments can be prohibitively expensive. The drugs often need to be given daily, which requires more personnel to handle the animals, and the technology used to image tumours in living animals can be costly. Mouse hospitals will probably have to invest heavily in generating trial-specific mouse models of cancer, cautions Tuveson, who has been using the approach to tackle pancreatic cancer. "You need the infrastructure to breed these animals and then to follow them just like you would in a hospital, and most laboratories do not have that."

But funders are aware of the approach's promise. In 2009, the US National Cancer Institute in Bethesda, Maryland, awarded US\$4.2 million to Pier Paolo Pandolfi, a cancer researcher at Harvard Medical School in Boston who is a co-author on the Nature study, to establish the co-clinical trial project in lung and prostate cancer. Additional grants from Stand Up To Cancer, a project of the Entertainment Industry Foundation charity, based in Los Angeles, California, are funding co-clinical trials in breast and ovarian cancer. It is a new way, says Tuveson, "to use mice as a looking glass into the human condition". - 